\title{
Association between IgG4-related disease and progressively transformed germinal centers of lymph nodes
}

Yasuharu Sato ${ }^{1}$, Dai Inoue ${ }^{2}$, Naoko Asano ${ }^{3}$, Katsuyoshi Takata ${ }^{1}$, Hideki Asaoku ${ }^{4}$, Yoshinobu Maeda ${ }^{5}$, Toshiaki Morito ${ }^{6}$, Hirokazu Okumura ${ }^{7}$, Shin Ishizawa ${ }^{8}$, Shoko Matsui ${ }^{9}$, Takayoshi Miyazono $^{10}$, Tamotsu Takeuchi ${ }^{11}$, Naoto Kuroda ${ }^{12}$, Yorihisa Orita ${ }^{13}$, Kiyoshi Takagawa $^{14}$, Masaru Kojima ${ }^{15}$ and Tadashi Yoshino ${ }^{1}$

${ }^{1}$ Department of Pathology, Okayama University Graduate School of Medicine, Dentistry and Pharmaceutical Sciences, Okayama, Japan; ${ }^{2}$ Department of Radiology, Kanazawa University Graduate School of Medical Science, Kanazawa, Japan; ${ }^{3}$ Department of Clinical Laboratory, Nagoya University Hospital, Nagoya, Japan; ${ }^{4}$ Department of Clinical Laboratory, Hiroshima Red Cross Hospital and Atomic-Bomb Survivors Hospital, Hiroshima, Japan; ${ }^{5}$ Department of Pathology, Toyama Red Cross Hospital, Toyama, Japan; ${ }^{6}$ Department of Anatomic Pathology, Kagawa Rosai Hospital, Marugame, Japan; ${ }^{7}$ Department of Internal Medicine, Toyama Prefectural Central Hospital, Toyama, Japan; ${ }^{8}$ Department of Pathology, Toyama Prefectural Central Hospital, Toyama, Japan; ${ }^{9}$ Department of First Internal Medicine, Graduate School of Medicine and Pharmaceutical

Science, University of Toyama, Toyama, Japan; ${ }^{10}$ Department of Gastroenterology and Hematology, Graduate School of Medicine and Pharmaceutical Science, University of Toyama, Toyama, Japan; ${ }^{11}$ Department of Pathology, Kochi Medical School, Kochi, Japan; ${ }^{12}$ Department of Diagnostic Pathology, Kochi Red Cross Hospital, Kochi, Japan; ${ }^{13}$ Department of Otolaryngology, Head and Neck Surgery, Okayama University Graduate School of Medicine, Dentistry and Pharmaceutical Sciences, Okayama, Japan; ${ }^{14}$ Department of Clinical Laboratory, Kurobe City Hospital, Kurobe, Japan and ${ }^{15}$ Department of Anatomic and Diagnostic Pathology, Dokkyo University School of Medicine, Mibu, Japan

Progressively transformed germinal centers is a benign condition of unknown pathogenesis characterized by a distinctive variant form of reactive follicular hyperplasia in lymph nodes. We recently reported Ig G4-related disease in progressively transformed germinal centers. However, no large case series has been reported and clinicopathologic findings remain unclear. Here, we report 40 Japanese patients (28 men, 12 women; median age, 56 years) with progressively transformed germinal centers of the lymph nodes who fulfilled the histological diagnostic criteria for IgG4-related disease (IgG4 ${ }^{+}$progressively transformed germinal centers), with asymptomatic localized lymphadenopathy involving the submandibular nodes in 24, submandibular and cervical nodes in 14, cervical nodes only in 1, and cervical and supraclavicular nodes in 1. In all, $16(52 \%)$ of 31 examined patients had allergic disease. Histologically, the lymph nodes demonstrated uniform histological findings, namely marked follicular hyperplasia with progressively transformed germinal centers, and localization of the majority of IgG4 ${ }^{+}$plasma cells in the germinal centers. Serum IgG4, serum IgE and peripheral blood eosinophils were elevated in $87 \%, 92 \%$ and $53 \%$ of examined patients, respectively. Eighteen patients subsequently developed extranodal lesions (including five who developed systemic disease), which on histological examination were consistent with IgG4-related disease. IgG4 ${ }^{+}$progressively transformed germinal centers presents with uniform clinicopathological features of asymptomatic localized submandibular lymphadenopathy, which persists and/or relapses, and sometimes progresses to extranodal lesions or systemic disease. Nine patients were administered steroid therapy when the lesions progressed, to which all

Correspondence: Dr Y Sato, MD, Department of Pathology, Okayama University Graduate School of Medicine, Dentistry, and Pharmaceutical Sciences, 2-5-1 Shikata-cho Kita-ku, Okayama 700-8558, Japan.

E-mail: satou-y@cc.okayama-u.ac.jp

Received 23 November 2011; revised 22 January 2012; accepted 22 January 2012; published online 6 April 2012 
responded well. We suggest that IgG4 ${ }^{+}$progressively transformed germinal centers should be included in the IgG4-related disease spectrum.

Modern Pathology (2012) 25, 956-967; doi:10.1038/modpathol.2012.54; published online 6 April 2012

Keywords: IgG4-related disease; IgG4-related lymphadenopathy; PTGC

The term progressively transformed germinal centers was first used by Lennert and Müller-Hermelink to describe reactive follicular hyperplasia in the lymph nodes, ${ }^{1}$ and progressively transformed germinal centers is observed in approximately $4 \%$ of patients with unspecific lymphadenopathy. ${ }^{1-6}$ Germinal centers in affected lymph nodes are usually larger than regular germinal centers and composed mainly of mantle zone lymphocytes and remnants of germinal center cells. Although relapse is frequent, affecting about $20 \%$ of patients, progressively transformed germinal centers is considered a non-malignant condition. ${ }^{2-6}$

IgG4-related disease is a recently recognized syndrome characterized by mass-forming lesions with lymphoplasmacytic infiltration, accumulation of $\mathrm{IgG} 4^{+}$plasma cells in affected tissues and increased serum IgG4 levels. ${ }^{7-14}$ IgG4-related disease generally involves either localized or systemic lymph nodes, ${ }^{8,13,14}$ and five histological subtypes of IgG4-related lymphadenopathy have been recognized. ${ }^{8}$ In 2009, we were the first to report patients with IgG4-related disease in progressively transformed germinal centers of the lymph nodes (progressively transformed germinal centers-type IgG4-related lymphadenopathy). ${ }^{14}$ Recently, while no large series has yet been reported, Grimm et al ${ }^{15}$ included 14 cases with progressively transformed germinal centers in their series. However, clinicopathologic findings remain unclear.

Here, we report the clinicopathological characteristics of 40 cases of progressively transformed germinal centers of the lymph nodes that fulfill the histological diagnostic criteria for IgG4-related disease.

\section{Materials and methods}

\section{Case Selection}

Two of us (YS and TY) reviewed the Pathology Department database of our institution using the search terms 'progressively transformed germinal centers,' 'follicular hyperplasia' and 'lymph node' for the 13-year period from 1998 to 2011. In all, 62 cases of progressively transformed germinal centers were identified, 40 of which fulfilled the histological diagnostic criteria of IgG4-related disease, namely the presence of IgG4 ${ }^{+}$plasma cells $>100$ / high-power fields (HPFs) and $\mathrm{IgG}^{+} / \mathrm{IgG}^{+}$plasma cell ratio $>40 \%$ (IgG4 ${ }^{+}$progressively transformed germinal centers) (Table 1), whereas 22 did not (IgG4- progressively transformed germinal centers).
The histological diagnostic criteria were outlined by the International Symposium on IgG4-RD (Boston, MA, USA, on 4-7 October 2011; http://www2.massgeneral.org/pathology/symposium/IgG4_related_systemic_dis.asp).

The clinical records and pathology materials of all cases were reviewed, and cases of multicentric Castleman's disease, malignant lymphoma or other lymphoproliferative disorders (including rheumatoid arthritis-related lymphadenopathy and other immune-mediated conditions, and so on) were histologically and clinically excluded.

\section{Histological Examination and Immunohistochemistry}

Surgically biopsied lymph node specimens were fixed in $10 \%$ formaldehyde and embedded in paraffin. Serial sections $(4 \mu \mathrm{m})$ were cut from each paraffin-embedded tissue block, and several sections were stained with hematoxylin and eosin. Immunohistochemistry was performed on paraffin sections using an automated Bond Max stainer (Leica Biosystems, Melbourne, Australia). The primary antibodies used were as follows: IgG (polyclonal (1:10 000); Dako), IgG4 (HP6025 (1:400); The Binding Site), Kappa (NCL-KAP (1:100); Novocastra) and Lambda (NCL-LAM (1:200); Novocastra). The number of IgG4 ${ }^{+}$or $\mathrm{IgG}^{+}$plasma cells was estimated for areas with the highest density of positive cells. Three different HPF $(\times 10$ in the eyepiece and $\times 40$ in the lens) in each section were counted, and the average number of positive cells per HPF was calculated.

\section{Polymerase Chain Reaction for the Detection of Ig Heavy-Chain Gene Rearrangement}

Ig heavy-chain gene rearrangement was analyzed by polymerase chain reaction performed according to standard procedures as described previously. ${ }^{14}$ The primers used for Ig heavy-chain gene amplification were $5^{\prime}$-TGG[A/G]TCCG[C/A]CAG[G/C]C[T/C][T/C] $\mathrm{C}[\mathrm{A} / \mathrm{C} / \mathrm{G} / \mathrm{T}] \mathrm{GG}-3^{\prime}$ as an upstream consensus V-region primer; $5^{\prime}$-TGAGGAGACGGTGACC-3' as a consensus J-region primer; and 5'-GTGACCAGGGT [A/C/G/T]CCTTGGCCCCAG-3' as a consensus J-region primer. ${ }^{14}$

\section{Statistical Analysis}

Differences in characteristics between the two groups were determined by the $\chi^{2}$ test, Fisher's 
Table 1 Clinical features of 40 patients with IgG4 ${ }^{+}$PTGC

\begin{tabular}{|c|c|c|c|c|c|c|c|c|c|c|}
\hline No. & Age/gender & $\begin{array}{l}\text { Biopsy site } \\
(\text { LN size }, \mathrm{cm})\end{array}$ & Initial presentation & Disease progression & $\begin{array}{l}\text { Treatment } \\
\text { (follow-up } \\
\text { period, } \\
\text { months) }\end{array}$ & $\begin{array}{l}\text { Allergic } \\
\text { disease }\end{array}$ & $\begin{array}{c}\text { Eosinophil } \\
\text { count in } P B \\
(\% ; n l<5 \%)\end{array}$ & $\begin{array}{c}I g G 4 \\
(\mathrm{mg} / \mathrm{dl} ; \\
n l=4.8-105)\end{array}$ & $\begin{array}{l}\operatorname{IgG4/IgG} \\
\quad(\% \\
n l=3-6)\end{array}$ & $\begin{array}{c}I g E \\
\text { (IU/ml; nl) }^{(I g E \text { ratio) }}\end{array}$ \\
\hline 1 & $36 / \mathrm{M}$ & $\begin{array}{l}\text { Submandibular LN } \\
(1.5)\end{array}$ & $\begin{array}{l}\text { Bil. submandibular } \\
\text { lymphadenopathy } \\
\text { and lt. submandibular } \\
\text { gland swelling }\end{array}$ & $\begin{array}{l}\text { None (but residual } \\
\text { lymph node lesions } \\
\text { persisted) }\end{array}$ & $\begin{array}{l}\text { Follow-up and } \\
\text { stable (18) }\end{array}$ & Drug allergy & 6.8 & 110 & 10.9 & NA \\
\hline 2 & $75 / \mathrm{M}$ & $\begin{array}{l}\text { Submandibular LN } \\
\text { (3) }\end{array}$ & $\begin{array}{l}\text { Bil. submandibular } \\
\text { and cervical } \\
\text { lymphadenopathy }\end{array}$ & NA & NA & NA & NA & NA & NA & NA \\
\hline 3 & $50 / \mathrm{M}$ & $\begin{array}{l}\text { Submandibular LN } \\
\text { (2) }\end{array}$ & $\begin{array}{l}\text { Bil. submandibular } \\
\text { and cervical } \\
\text { lymphadenopathy }\end{array}$ & $\begin{array}{l}\text { Residual lymph node } \\
\text { lesions persisted and } \\
\text { patient developed bil. } \\
\text { axillary } \\
\text { lymphadenopathy } 3 \\
\text { years later }\end{array}$ & Follow-up (18) & $\begin{array}{l}\text { Allergic } \\
\text { rhinitis }\end{array}$ & 21.1 & 183 & 6.74 & NA \\
\hline 4 & $50 / \mathrm{F}$ & $\begin{array}{l}\text { Submandibular LN } \\
(1.5)\end{array}$ & $\begin{array}{l}\text { Bil. submandibular } \\
\text { lymphadenopathy }\end{array}$ & $\begin{array}{l}\text { None (but residual } \\
\text { lymph node lesions } \\
\text { persisted) }\end{array}$ & $\begin{array}{l}\text { Follow-up and } \\
\text { stable (4) }\end{array}$ & $\begin{array}{l}\text { Allergic } \\
\text { rhinitis }\end{array}$ & 2 & 24 & 2 & NA \\
\hline 5 & $66 / \mathrm{M}$ & $\begin{array}{l}\text { Submandibular LN } \\
\text { (2) }\end{array}$ & $\begin{array}{l}\text { Lt. submandibular } \\
\text { lymphadenopathy }\end{array}$ & $\begin{array}{l}\text { Relapsed lt. } \\
\text { submandibular } \\
\text { lymphadenopathy } 10 \\
\text { months later }\end{array}$ & Follow-up (10) & None & 5.9 & $314^{\mathrm{c}}$ & $19.2^{\mathrm{c}}$ & $505(2.9)$ \\
\hline 6 & $46 / \mathrm{M}$ & $\begin{array}{l}\text { Submandibular LN } \\
\text { (3.5) }\end{array}$ & $\begin{array}{l}\text { Lt. submandibular } \\
\text { lymphadenopathy, lt. } \\
\text { submandibular gland } \\
\text { swelling and } \\
\text { thickened rt. pleura }\end{array}$ & $\begin{array}{l}\text { None (but residual } \\
\text { lymph node lesions } \\
\text { persisted) }\end{array}$ & $\begin{array}{l}\text { Follow-up and } \\
\text { stable (10) }\end{array}$ & NA & 3 & NA & NA & NA \\
\hline 7 & $71 / \mathrm{M}$ & $\begin{array}{l}\text { Submandibular LN } \\
(1.5)\end{array}$ & $\begin{array}{l}\text { Lt. submandibular } \\
\text { and cervical } \\
\text { lymphadenopathy }\end{array}$ & $\begin{array}{l}\text { None (but residual } \\
\text { lymph node lesions } \\
\text { persisted) }\end{array}$ & $\begin{array}{l}\text { Follow-up and } \\
\text { stable (5) }\end{array}$ & None & 2.5 & 275 & 12.8 & $259(1.5)$ \\
\hline 8 & $62 / \mathrm{F}$ & $\begin{array}{l}\text { Submandibular LN } \\
\text { (1.5) }\end{array}$ & $\begin{array}{l}\text { Lt. submandibular } \\
\text { lymphadenopathy }\end{array}$ & None & $\begin{array}{l}\text { Follow-up and } \\
\text { stable (6) }\end{array}$ & $\begin{array}{l}\text { Contact } \\
\text { dermatitis }\end{array}$ & 0 & NA & NA & NA \\
\hline 9 & $75 / \mathrm{F}$ & $\begin{array}{l}\text { Cervical and } \\
\text { supraclavicular LN } \\
\text { (2) }\end{array}$ & $\begin{array}{l}\text { Rt. cervical and } \\
\text { supraclavicular } \\
\text { lymphadenopathy }\end{array}$ & $\begin{array}{l}\text { None (the cervical LN } \\
\text { was biopsied } 9 \\
\text { months ago but a } \\
\text { residual } \\
\text { supraclavicular } \\
\text { lymph node lesion } \\
\text { persisted) }\end{array}$ & $\begin{array}{l}\text { Follow-up and } \\
\text { stable (10) }\end{array}$ & Food allergy & 8 & 36.2 & 2.3 & $47.0(0.27)$ \\
\hline 10 & $45 / \mathrm{F}$ & Cervical LN (3) & $\begin{array}{l}\text { Lt. cervical } \\
\text { lymphadenopathy }\end{array}$ & $\begin{array}{l}\text { Residual lymph node } \\
\text { lesions persisted; LN } \\
\text { size increased in } 3 \\
\text { years }\end{array}$ & Follow-up (36) & $\begin{array}{l}\text { Asthma and } \\
\text { drug allergy }\end{array}$ & 7 & NA & NA & NA \\
\hline 11 & $64 / \mathrm{M}$ & $\begin{array}{l}\text { Submandibular LN } \\
\text { (3) }\end{array}$ & $\begin{array}{l}\text { Rt. submandibular } \\
\text { lymphadenopathy }\end{array}$ & $\begin{array}{l}\text { Relapsed rt. } \\
\text { submandibular } \\
\text { lymphadenopathy } \\
2 \text { years later; patient } \\
\text { developed bil. } \\
\text { lacrimal, } \\
\text { submandibular gland } \\
\text { swelling, mediastinal } \\
\text { lymphadenopathy, } \\
\text { and kidney lesion } \\
\text { 11 years later }\end{array}$ & $\begin{array}{l}\text { Steroid } \\
\text { therapy was } \\
\text { performed } \\
\text { when disease } \\
\text { progressed, } \\
\text { with good } \\
\text { response (144) }\end{array}$ & $\begin{array}{l}\text { Asthma, } \\
\text { drug allergy } \\
\text { and allergic } \\
\text { rhinitis }\end{array}$ & 10.5 & $2550^{\mathrm{c}}$ & $42.3^{\mathrm{c}}$ & NA \\
\hline 12 & $60 / \mathrm{M}$ & $\begin{array}{l}\text { Submandibular LN } \\
\text { (2) }\end{array}$ & $\begin{array}{l}\text { Lt. submandibular } \\
\text { and cervical } \\
\text { lymphadenopathy }\end{array}$ & $\mathrm{NA}$ & NA & None & 2.2 & NA & NA & NA \\
\hline
\end{tabular}


Table 1 Continued

\begin{tabular}{|c|c|c|c|c|c|c|c|c|c|c|}
\hline No. & Age/gender & $\begin{array}{l}\text { Biopsy site } \\
(L N \text { size }, \mathrm{cm})\end{array}$ & Initial presentation & Disease progression & $\begin{array}{l}\text { Treatment } \\
\text { (follow-up } \\
\text { period, } \\
\text { months) }\end{array}$ & $\begin{array}{l}\text { Allergic } \\
\text { disease }\end{array}$ & $\begin{array}{l}\text { Eosinophil } \\
\text { count in PB } \\
(\% ; n l<5 \%)\end{array}$ & $\begin{array}{c}\operatorname{IgG4} \\
(\mathrm{mg} / \mathrm{dl} ; \\
n l=4.8-105)\end{array}$ & $\begin{array}{c}\operatorname{IgG4/IgG} \\
\quad(\% \\
n l=3-6)\end{array}$ & $\begin{array}{c}I g E \\
(\mathrm{IU} / \mathrm{ml} ; \mathrm{nl}) \\
{\text { (IgE ratio })^{\mathrm{a}}}\end{array}$ \\
\hline 13 & $61 / \mathrm{M}$ & $\begin{array}{l}\text { Submandibular LN } \\
\text { (2) }\end{array}$ & $\begin{array}{l}\text { Lt. submandibular } \\
\text { lymphadenopathy }\end{array}$ & $\begin{array}{l}\text { Developed bil. } \\
\text { submandibular gland } \\
\text { swelling, } \\
\text { mediastinum, lung, } \\
\text { pancreas kidney and } \\
\text { aortic lesions } 3 \text { years } \\
\text { later }\end{array}$ & $\begin{array}{l}\text { Steroid } \\
\text { therapy was } \\
\text { performed } \\
\text { when disease } \\
\text { progressed, } \\
\text { with good } \\
\text { response (101) }\end{array}$ & None & 4 & $2240^{\mathrm{c}}$ & $77.5^{\mathrm{c}}$ & NA \\
\hline 14 & $43 / \mathrm{F}$ & $\begin{array}{l}\text { Submandibular LN } \\
\text { (1) }\end{array}$ & $\begin{array}{l}\text { Rt. submandibular } \\
\text { and cervical } \\
\text { lymphadenopathy }\end{array}$ & NA & NA & NA & NA & NA & NA & NA \\
\hline 15 & $46 / \mathrm{M}$ & $\begin{array}{l}\text { Submandibular LN } \\
\text { (3) }\end{array}$ & $\begin{array}{l}\text { Lt. submandibular } \\
\text { lymphadenopathy }\end{array}$ & $\begin{array}{l}\text { Relapsed lt. } \\
\text { submandibular LN } 5 \\
\text { years later }{ }^{\mathrm{b}} \text { Relapsed } \\
\text { rt. submandibular LN } \text { 8 years later. }\end{array}$ & $\begin{array}{l}\text { Follow-up } \\
(100)\end{array}$ & None & 7.7 & 40 & 2.6 & $681(1.9)$ \\
\hline 16 & $58 / \mathrm{F}$ & $\begin{array}{l}\text { Submandibular LN } \\
(1.5)\end{array}$ & $\begin{array}{l}\text { Rt. submandibular } \\
\text { lymphadenopathy }\end{array}$ & $\begin{array}{l}\text { Developed bil. } \\
\text { submandibular gland } \\
\text { swelling } 1 \text { year later } \\
\text { and bil. lacrimal gland } \\
\text { swelling } 4 \text { years later }\end{array}$ & Follow-up (48) & $\begin{array}{l}\text { Contact } \\
\text { dermatitis }\end{array}$ & 2.2 & $241^{\mathrm{c}}$ & $18.4^{\mathrm{c}}$ & $280^{\circ}(1.6)$ \\
\hline 17 & $55 / \mathrm{M}$ & $\begin{array}{l}\text { Submandibular LN } \\
\text { (3) }\end{array}$ & $\begin{array}{l}\text { Rt. submandibular } \\
\text { and cervical } \\
\text { lymphadenopathy }\end{array}$ & $\begin{array}{l}\text { None (but residual } \\
\text { lymph node lesions } \\
\text { persisted) }\end{array}$ & $\begin{array}{l}\text { Follow-up and } \\
\text { stable (6) }\end{array}$ & None & 4.1 & NA & NA & NA \\
\hline 18 & $52 / \mathrm{F}$ & $\begin{array}{l}\text { Submandibular LN } \\
(2.5)\end{array}$ & $\begin{array}{l}\text { Lt. submandibular } \\
\text { and cervical } \\
\text { lymphadenopathy }\end{array}$ & $\begin{array}{l}\text { None (but residual } \\
\text { lymph node lesions } \\
\text { persisted) }\end{array}$ & $\begin{array}{l}\text { Follow-up and } \\
\text { stable (3) }\end{array}$ & NA & 4 & NA & NA & NA \\
\hline 19 & $51 / \mathrm{F}$ & $\begin{array}{l}\text { Submandibular LN } \\
\text { (2) }\end{array}$ & $\begin{array}{l}\text { Rt. submandibular } \\
\text { lymphadenopathy }\end{array}$ & $\begin{array}{l}\text { Developed bil. } \\
\text { submandibular } \\
\text { lymphadenopathy } \\
\text { and rt. parotid gland } \\
\text { swelling } 1 \text { year later }\end{array}$ & Follow-up (18) & None & NA & 224 & NA & NA \\
\hline 20 & $52 / \mathrm{M}$ & $\begin{array}{l}\text { Submandibular LN } \\
(2.5)\end{array}$ & $\begin{array}{l}\text { Lt. submandibular } \\
\text { lymphadenopathy }\end{array}$ & $\begin{array}{l}\text { Developed bil. } \\
\text { lacrimal gland } \\
\text { swelling, skin lesion } \\
\text { and systemic } \\
\text { lymphadenopathy } 5 \\
\text { years later }{ }^{\mathrm{b}}\end{array}$ & $\begin{array}{l}\text { Steroid } \\
\text { therapy was } \\
\text { performed } \\
\text { when disease } \\
\text { progressed, } \\
\text { with good } \\
\text { response. } \\
\text { However, the } \\
\text { lesion } \\
\text { relapsed } 3 \\
\text { months later } \\
\text { (96) }\end{array}$ & None & $14^{\mathrm{c}}$ & $1700^{\mathrm{c}}$ & $37.5^{\mathrm{c}}$ & $904^{\mathrm{c}}(2.5)$ \\
\hline 21 & $43 / \mathrm{M}$ & $\begin{array}{l}\text { Submandibular LN } \\
\text { (2) }\end{array}$ & $\begin{array}{l}\text { Lt. submandibular } \\
\text { lymphadenopathy }\end{array}$ & $\begin{array}{l}\text { Developed bil. } \\
\text { lacrimal gland } \\
\text { swelling, skin lesion } \\
\text { and systemic } \\
\text { lymphadenopathy } 3 \\
\text { years later }\end{array}$ & $\begin{array}{l}\text { Steroid } \\
\text { therapy was } \\
\text { performed } \\
\text { when disease } \\
\text { progressed, } \\
\text { with good } \\
\text { response. } \\
\text { However, the } \\
\text { lesion } \\
\text { relapsed } 3 \\
\text { years later (78) }\end{array}$ & $\begin{array}{l}\text { Atopic } \\
\text { dermatitis }\end{array}$ & 9 & $216^{\mathrm{c}}$ & $13.72^{\mathrm{c}}$ & $1550^{\mathrm{c}}(4.3)$ \\
\hline
\end{tabular}


Table 1 Continued

\begin{tabular}{|c|c|c|c|c|c|c|c|c|c|c|}
\hline No. & Age/gender & $\begin{array}{l}\text { Biopsy site } \\
(L N \text { size, } \mathrm{cm})\end{array}$ & Initial presentation & Disease progression & $\begin{array}{l}\text { Treatment } \\
\text { (follow-up } \\
\text { period, } \\
\text { months) }\end{array}$ & $\begin{array}{l}\text { Allergic } \\
\text { disease }\end{array}$ & $\begin{array}{c}\text { Eosinophil } \\
\text { count in } P B \\
(\% ; n l<5 \%)\end{array}$ & $\begin{array}{c}I g G 4 \\
(m g / d l ; \\
n l=4.8-105)\end{array}$ & $\begin{array}{l}I g G 4 / \operatorname{IgG} \\
\quad(\% \\
n l=3-6)\end{array}$ & $\begin{array}{c}I g E \\
(\mathrm{IU} / \mathrm{ml} ; \mathrm{nl}) \\
{\text { (IgE ratio })^{\mathrm{a}}}\end{array}$ \\
\hline 22 & $58 / \mathrm{M}$ & $\begin{array}{l}\text { Submandibular LN } \\
\text { (2) }\end{array}$ & $\begin{array}{l}\text { Bil. submandibular } \\
\text { and cervical } \\
\text { lymphadenopathy }\end{array}$ & $\begin{array}{l}\text { Developed prostatic } \\
\text { lesion and systemic } \\
\text { lymphadenopathy } 2 \\
\text { years later }^{\mathrm{b}}\end{array}$ & $\begin{array}{l}\text { Steroid } \\
\text { therapy was } \\
\text { performed } \\
\text { when disease } \\
\text { progressed, } \\
\text { with good } \\
\text { response. } \\
\text { However, the } \\
\text { lesion } \\
\text { relapsed } 10 \\
\text { months later } \\
\text { (39) }\end{array}$ & Drug allergy & 8 & $1280^{\mathrm{c}}$ & $30.74^{\mathrm{c}}$ & $641^{\mathrm{c}}(1.8)$ \\
\hline 23 & $50 / \mathrm{M}$ & $\begin{array}{l}\text { Submandibular LN } \\
\text { (1) }\end{array}$ & $\begin{array}{l}\text { Bil. submandibular } \\
\text { lymphadenopathy }\end{array}$ & None & $\begin{array}{l}\text { Follow-up and } \\
\text { stable (36) }\end{array}$ & None & 4 & NA & NA & NA \\
\hline 24 & $45 / \mathrm{F}$ & $\begin{array}{l}\text { Submandibular LN } \\
(2.5)\end{array}$ & $\begin{array}{l}\text { Rt. submandibular } \\
\text { and cervical } \\
\text { lymphadenopathy }\end{array}$ & $\begin{array}{l}\text { Developed bil. } \\
\text { lacrimal gland } \\
\text { swelling } 1 \text { year later }\end{array}$ & $\begin{array}{l}\text { Steroid } \\
\text { therapy was } \\
\text { performed } \\
\text { when disease } \\
\text { progressed, } \\
\text { with good } \\
\text { response (16) }\end{array}$ & None & 4 & NA & NA & $800(2.2)$ \\
\hline 25 & $67 / \mathrm{M}$ & $\begin{array}{l}\text { Submandibular LN } \\
\text { (3) }\end{array}$ & $\begin{array}{l}\text { Bil. submandibular } \\
\text { lymphadenopathy }\end{array}$ & $\begin{array}{l}\text { None (but residual } \\
\text { lymph node lesions } \\
\text { persisted) }\end{array}$ & $\begin{array}{l}\text { Follow-up and } \\
\text { stable }(72)\end{array}$ & NA & 8.2 & NA & NA & NA \\
\hline 26 & $51 / \mathrm{F}$ & $\begin{array}{l}\text { Submandibular LN } \\
(3.5)\end{array}$ & $\begin{array}{l}\text { Rt. submandibular } \\
\text { lymphadenopathy } \\
\text { and rt. submandibular } \\
\text { gland swelling }\end{array}$ & None & $\begin{array}{l}\text { Follow-up and } \\
\text { stable (12) }\end{array}$ & None & 2.8 & NA & NA & NA \\
\hline 27 & $42 / \mathrm{M}$ & $\begin{array}{l}\text { Submandibular LN } \\
\text { (2) }\end{array}$ & $\begin{array}{l}\text { Lt. submandibular } \\
\text { and cervical } \\
\text { lymphadenopathy }\end{array}$ & $\begin{array}{l}\text { None (but residual } \\
\text { lymph node lesions } \\
\text { persisted) }\end{array}$ & $\begin{array}{l}\text { Follow-up and } \\
\text { stable (60) }\end{array}$ & None & 6.1 & NA & NA & $241(1.4)$ \\
\hline 28 & 49/M & $\begin{array}{l}\text { Submandibular LN } \\
(2.5)\end{array}$ & $\begin{array}{l}\text { Lt. submandibular, } \\
\text { cervical } \\
\text { lymphadenopathy, } \\
\text { and lt. submandibular } \\
\text { gland swelling }\end{array}$ & NA & NA & NA & 1 & NA & NA & NA \\
\hline 29 & $58 / \mathrm{M}$ & $\begin{array}{l}\text { Submandibular LN } \\
(2.5)\end{array}$ & $\begin{array}{l}\text { Rt. submandibular } \\
\text { lymphadenopathy }\end{array}$ & $\begin{array}{l}\text { Developed bil. } \\
\text { lacrimal gland } \\
\text { swelling and rt. } \\
\text { maxillary sinus tumor } \\
3 \text { years later }\end{array}$ & $\begin{array}{l}\text { Steroid } \\
\text { therapy was } \\
\text { performed } \\
\text { when disease } \\
\text { progressed, } \\
\text { with good } \\
\text { response (39) }\end{array}$ & $\begin{array}{l}\text { Allergic } \\
\text { rhinitis }\end{array}$ & 9 & $921^{\mathrm{c}}$ & $47.5^{\mathrm{c}}$ & $1090^{\circ}(3.0)$ \\
\hline 30 & $60 / \mathrm{M}$ & $\begin{array}{l}\text { Submandibular LN } \\
(2.5)\end{array}$ & $\begin{array}{l}\text { Rt. submandibular } \\
\text { lymphadenopathy }\end{array}$ & None & $\begin{array}{l}\text { Follow-up and } \\
\text { stable (26) }\end{array}$ & Asthma & 3 & NA & NA & NA \\
\hline 31 & $46 / \mathrm{F}$ & $\begin{array}{l}\text { Submandibular LN } \\
\text { (2) }\end{array}$ & $\begin{array}{l}\text { Rt. submandibular } \\
\text { lymphadenopathy } \\
\text { and rt. submandibular } \\
\text { gland swelling }\end{array}$ & NA & NA & $\begin{array}{l}\text { Allergic } \\
\text { rhinitis }\end{array}$ & NA & NA & NA & NA \\
\hline 32 & $72 / \mathrm{M}$ & $\begin{array}{l}\text { Submandibular LN } \\
(2.5)\end{array}$ & $\begin{array}{l}\text { Bil. submandibular } \\
\text { lymphadenopathy }\end{array}$ & $\begin{array}{l}\text { None (but residual } \\
\text { lymph node lesions } \\
\text { persisted) }\end{array}$ & $\begin{array}{l}\text { Follow-up and } \\
\text { stable (12) }\end{array}$ & NA & 0.4 & NA & NA & NA \\
\hline
\end{tabular}


Table 1 Continued

\begin{tabular}{|c|c|c|c|c|c|c|c|c|c|c|}
\hline No. & Age/gender & $\begin{array}{l}\text { Biopsy site } \\
(\text { LN size, } \mathrm{cm})\end{array}$ & Initial presentation & Disease progression & $\begin{array}{l}\text { Treatment } \\
\text { (follow-up } \\
\text { period, } \\
\text { months) }\end{array}$ & $\begin{array}{l}\text { Allergic } \\
\text { disease }\end{array}$ & $\begin{array}{l}\text { Eosinophil } \\
\text { count in } P B \\
(\% ; n l<5 \%)\end{array}$ & $\begin{array}{c}\operatorname{IgG4} \\
(\mathrm{mg} / \mathrm{dl} ; \\
n l=4.8-105)\end{array}$ & $\begin{array}{c}I g G 4 / I g G \\
\quad(\% \\
n l=3-6)\end{array}$ & $\begin{array}{c}I g E \\
(I U / m l ; n l) \\
\left(_{(I g E ~ r a t i o)^{\mathrm{a}}}\right.\end{array}$ \\
\hline 33 & $51 / \mathrm{M}$ & $\begin{array}{l}\text { Submandibular LN } \\
(3.5)\end{array}$ & $\begin{array}{l}\text { Rt. submandibular } \\
\text { and cervical } \\
\text { lymphadenopathy }\end{array}$ & $\begin{array}{l}\text { None (but residual } \\
\text { lymph node lesions } \\
\text { persisted) }\end{array}$ & $\begin{array}{l}\text { Follow-up and } \\
\text { stable (26) }\end{array}$ & $\begin{array}{l}\text { Allergic } \\
\text { rhinitis }\end{array}$ & 6.7 & 169 & 7 & $3250(18.8)$ \\
\hline 34 & $68 / \mathrm{F}$ & $\begin{array}{l}\text { Submandibular LN } \\
\text { (1.5) }\end{array}$ & $\begin{array}{l}\text { Rt. submandibular } \\
\text { lymphadenopathy } \\
\text { and rt. lacrimal gland } \\
\text { swelling }\end{array}$ & $\begin{array}{l}\text { None (but residual } \\
\text { lymph node lesions } \\
\text { persisted) }\end{array}$ & $\begin{array}{l}\text { Follow-up and } \\
\text { stable (25) }\end{array}$ & $\begin{array}{l}\text { Food and } \\
\text { drug allergy }\end{array}$ & 5.6 & NA & NA & NA \\
\hline 35 & $67 / \mathrm{M}$ & $\begin{array}{l}\text { Submandibular LN } \\
(2)\end{array}$ & $\begin{array}{l}\text { Lt. submandibular } \\
\text { lymphadenopathy }\end{array}$ & None & $\begin{array}{l}\text { Follow-up and } \\
\text { stable (27) }\end{array}$ & $\begin{array}{l}\text { Allergic } \\
\text { rhinitis and } \\
\text { asthma }\end{array}$ & NA & NA & NA & NA \\
\hline 36 & $70 / \mathrm{M}$ & $\begin{array}{l}\text { Submandibular LN } \\
\text { (2) }\end{array}$ & $\begin{array}{l}\text { Lt. submandibular } \\
\text { lymphadenopathy } \\
\text { and lt. parotid gland } \\
\text { tumor }\end{array}$ & $\begin{array}{l}\text { Developed rt. } \\
\text { submandibular gland } \\
\text { swelling and } \\
\text { pancreatic lesion } \\
2 \text { years later }\end{array}$ & $\begin{array}{l}\text { Steroid } \\
\text { therapy was } \\
\text { performed } \\
\text { when disease } \\
\text { progressed, } \\
\text { with good } \\
\text { response (43) }\end{array}$ & NA & 10.9 & $483^{\mathrm{c}}$ & $27.7^{\mathrm{c}}$ & NA \\
\hline 37 & $51 / \mathrm{M}$ & $\begin{array}{l}\text { Submandibular LN } \\
\text { (1.5) }\end{array}$ & $\begin{array}{l}\text { Rt. submandibular, } \\
\text { cervical } \\
\text { lymphadenopathy, } \\
\text { and bil. } \\
\text { submandibular gland } \\
\text { swelling }\end{array}$ & $\begin{array}{l}\text { None (but residual } \\
\text { lymph node lesions } \\
\text { persisted) }\end{array}$ & $\begin{array}{l}\text { Follow-up and } \\
\text { stable (18) }\end{array}$ & None & 6.3 & NA & NA & NA \\
\hline 38 & $61 / \mathrm{M}$ & $\begin{array}{l}\text { Submandibular LN } \\
\text { (2) }\end{array}$ & $\begin{array}{l}\text { Bil. submandibular } \\
\text { and cervical } \\
\text { lymphadenopathy }\end{array}$ & NA & NA & NA & 2.6 & NA & NA & NA \\
\hline 39 & $76 / \mathrm{M}$ & $\begin{array}{l}\text { Submandibular LN } \\
(1.5)\end{array}$ & $\begin{array}{l}\text { Rt. submandibular } \\
\text { lymphadenopathy }\end{array}$ & $\begin{array}{l}\text { Developed skin } \\
\text { lesion }^{\mathrm{b}} \text { and bil. } \\
\text { lacrimal gland } \\
\text { swelling } 2 \text { years later }\end{array}$ & $\begin{array}{l}\text { Steroid } \\
\text { therapy was } \\
\text { performed on } \\
\text { bil. swelling of } \\
\text { the lacrimal } \\
\text { gland, with } \\
\text { good response } \\
\text { (63) }\end{array}$ & Asthma & 11.6 & NA & NA & $875(2.4)$ \\
\hline 40 & $57 / \mathrm{M}$ & $\begin{array}{l}\text { Submandibular LN } \\
\text { (3) }\end{array}$ & $\begin{array}{l}\text { Rt. submandibular } \\
\text { lymphadenopathy }\end{array}$ & $\begin{array}{l}\text { Relapsed rt. } \\
\text { submandibular } \\
\text { lymphadenopathy } 2 \\
\text { and } 8 \text { years later }\end{array}$ & Follow-up (97) & None & NA & NA & NA & NA \\
\hline
\end{tabular}

Abbreviations: Bil., bilateral; LN, lymph node; lt., left; NA, not available; nl, normal; PB, peripheral blood; PTGC, progressively transformed germinal centers; rt., right.

${ }^{\mathrm{a}} \mathrm{IgE}$ ratio: measured value/normal value.

$\mathrm{b}^{\mathrm{b}}$ The lesion was histologically diagnosed as IgG4-related disease.

${ }^{\mathrm{C}}$ The data was obtained at relapse or disease progression time. 
Table 2 Summary of clinical features of IgG4 ${ }^{+}$PTGC

\begin{tabular}{|c|c|}
\hline \multirow{2}{*}{\multicolumn{2}{|c|}{$\begin{array}{l}\text { Number } \\
\text { Gender }\end{array}$}} \\
\hline & \\
\hline Male/female & $28 / 12$ \\
\hline \multicolumn{2}{|l|}{ Age } \\
\hline Median (range) & $56(36-76)$ \\
\hline$\geq 60$ & $16(40 \%)$ \\
\hline Allergic disease history & $16 / 31(51.6 \%)$ \\
\hline \multicolumn{2}{|l|}{ Laboratory findings } \\
\hline $\begin{array}{l}\text { Increased eosinophil count in peripheral } \\
\text { blood }\end{array}$ & $18 / 34(52.9 \%)$ \\
\hline Elevated serum IgG4 level & $14 / 17(82.4 \%)$ \\
\hline Elevated serum IgE level & $12 / 13(92.3 \%)$ \\
\hline \multicolumn{2}{|l|}{ Initial lymphadenopathy } \\
\hline Submandibular lymphadenopathy & 24 (bilateral; 5) \\
\hline Submandibular and cervical & 14 (bilateral; 4) \\
\hline $\begin{array}{l}\text { lymphadenopathy } \\
\text { Cervical lymphadenopathy }\end{array}$ & 1 (unilateral) \\
\hline $\begin{array}{l}\text { Cervical and supraclavicular } \\
\text { lymphadenopathy }\end{array}$ & 1 (unilateral) \\
\hline Number of available follow-up reports & 34 \\
\hline \multicolumn{2}{|l|}{ Follow-up period } \\
\hline Median (range) & $26(3-144)$ \\
\hline Persistence or relapse of lymph node lesions & $23 / 34(67.6 \%)$ \\
\hline Progression to extranodal lesions & $18 / 34(52.9 \%)$ \\
\hline Submandibular gland & 5 \\
\hline Lacrimal gland & 2 \\
\hline Lacrimal gland and submandibular gland & 1 \\
\hline Lacrimal gland and skin & 3 \\
\hline Submandibular gland and pleura & 1 \\
\hline Lacrimal gland and maxillary sinus & 1 \\
\hline $\begin{array}{l}\text { Lacrimal gland, submandibular gland, } \\
\text { parotid gland, mediastinum, kidney }\end{array}$ & 1 \\
\hline $\begin{array}{l}\text { Submandibular gland, mediastinum, lung, } \\
\text { pancreas, aorta, kidney }\end{array}$ & 1 \\
\hline Submandibular gland and parotid gland & 1 \\
\hline Parotid gland & 1 \\
\hline Prostate & 1 \\
\hline Progression to systemic lymphadenopathy & $3 / 34(8.8 \%)$ \\
\hline
\end{tabular}

Abbreviation: PTGC, progressively transformed germinal centers. exact test, Student's $t$-test or Mann-Whitney $U$-test, as appropriate. All data were analyzed with the STATA software (version 10.0; Stata, College Station, TX, USA).

\section{Results}

\section{Clinical Features of IgG4 ${ }^{+}$Progressively Transformed Germinal Centers}

Clinical findings are summarized in Tables 1 and 2 . There were 28 men and 12 women with a median age of 56 years (range, 36-76 years). On initial clinical examination, all patients presented with localized submandibular and/or cervical, or cervical and supraclavicular lymphadenopathy. Twenty-four patients showed submandibular lymphadenopathy, which was bilateral in five. Fourteen patients showed submandibular and cervical lymphadenopathy, which was bilateral in four. Only two patients showed cervical, or cervical and supraclavicular lymphadenopathy. In total, 38 (95\%) of 40 patients showed submandibular lymphadenopathy. Lymph node biopsy revealed that the size of the biopsied lymph nodes ranged from 1 to $3.5 \mathrm{~cm}$ in diameter, with an average of $2.2 \mathrm{~cm}$. In addition, ${ }^{18} \mathrm{~F}$-fluorodeoxy glucose positron emission tomography showed significantly elevated uptake in examined patients (Figure 1). The lesions were therefore all suspected to be malignant lymphomas at initial clinical diagnosis.

Among patients examined for each respective factor, allergic disease was identified in 16 (52\%) of 31 patients; peripheral blood eosinophil count was increased in $18(53 \%)$ of 34 ; serum IgG4 levels were elevated in $14(82 \%)$ of 17 ; and serum $\operatorname{IgE}$ levels were elevated in $12(92 \%)$ of 13.
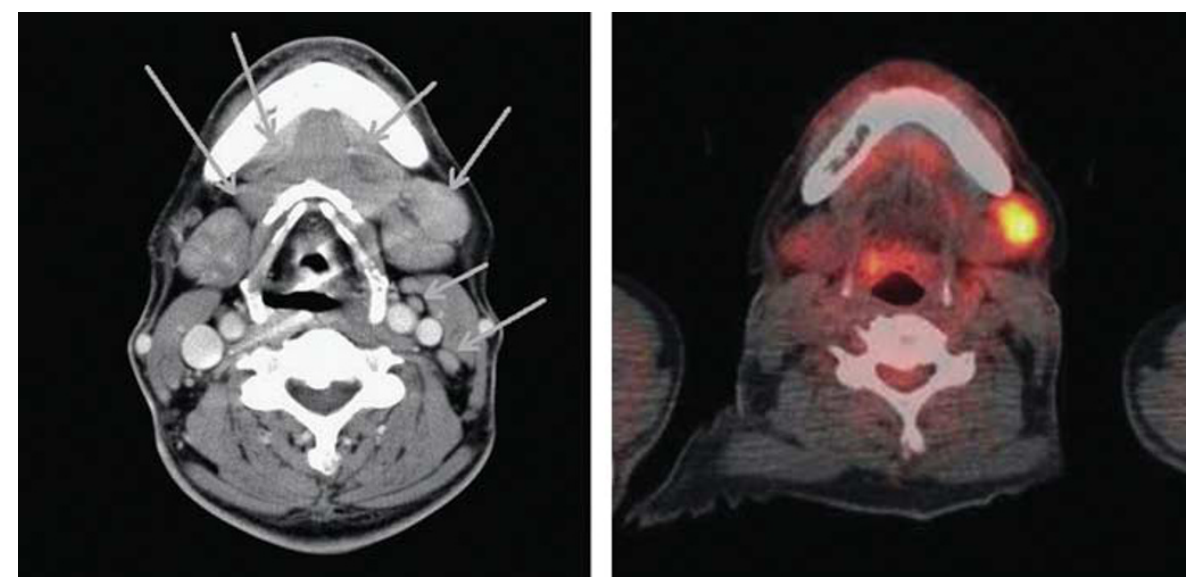

Figure 1 Ragiological images of IgG4 ${ }^{+}$progressively transformed germinal centers. Patient no. 12 had localized left submandibular lymphadenopathy and cervical lymphadenopathy (left panel). ${ }^{18} \mathrm{~F}$-fluorodeoxy glucose positron emission tomography showed significantly elevated uptake in the left submandibular lymph node (right panel). The lesion was radiologically and clinically suspected to be malignant lymphoma. 
Table 3 Clinicopathological characteristics of patients with IgG4 ${ }^{+}$PTGC and IgG4 ${ }^{-}$PTGC

\begin{tabular}{|c|c|c|c|}
\hline & $\begin{array}{c}I g G 4^{+} P T G C \\
(\mathrm{n}=40)\end{array}$ & 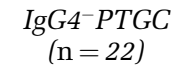 & $\mathrm{P}^{\mathrm{a}}$ \\
\hline \multicolumn{4}{|l|}{ Gender } \\
\hline (Male/female) & $28 / 12$ & $13 / 9$ & 0.39 \\
\hline \multicolumn{4}{|l|}{ Age (years) } \\
\hline Median & 56 & 47 & 0.060 \\
\hline Mean (range) & $56.5(36-76)$ & $46.6(18-78)$ & \\
\hline$>40$ years old & $39(98 \%)$ & $14(64 \%)$ & $<0.0001$ \\
\hline$>50$ years old & $30(75 \%)$ & $10(45 \%)$ & 0.02 \\
\hline \multicolumn{4}{|c|}{ Lymphadenopathy area } \\
\hline $\begin{array}{l}\text { Submandibular } \\
\text { LN }\end{array}$ & $38(95 \%)$ & $1(4.5 \%)$ & $<0.0001$ \\
\hline Cervical LN & $16(40 \%)$ & $11(50 \%)$ & 0.45 \\
\hline $\begin{array}{l}\text { Supraclavicular } \\
\text { LN }\end{array}$ & $1(2.5 \%)$ & $1(4.6 \%)$ & 0.66 \\
\hline Axillary LN & $0(0 \%)$ & $6(27 \%)$ & 0.001 \\
\hline Paraaortic LN & $0(0 \%)$ & $2(9 \%)$ & 0.053 \\
\hline Inguinal LN & $0(0 \%)$ & $5(23 \%)$ & 0.002 \\
\hline \multicolumn{4}{|c|}{ IgG4 $4^{+} / \operatorname{Ig} G^{+}$plasma cell ratio } \\
\hline Mean & 57.4 & 5.0 & \\
\hline Median (range) & $57.8(44.2-78.1)$ & $3.7(0-16.7)$ & $<0.0001$ \\
\hline
\end{tabular}

Abbreviations: LN, lymph node; PTGC, progressively transformed germinal centers.

${ }^{\mathrm{a}} \mathrm{IgG} 4{ }^{+}$PTGC vs IgG4 ${ }^{-}$PTGC.

Clinicopathological Differences Between IgG4 ${ }^{+}$ Progressively Transformed Germinal Centers and IgG4 ${ }^{-}$Progressively Transformed Germinal Centers

The clinicopathological findings associated with 40 cases of $\mathrm{IgG}^{+}$progressively transformed germinal centers and 22 of IgG4- progressively transformed germinal centers $\left(\mathrm{IgG}^{+} / \mathrm{IgG}^{+}\right.$plasma cell ratio $\leq 40 \%$ ) are summarized in Table 3 , Supplementary Figure 1 and Supplementary Table 1. Lymph nodes affected by $\mathrm{IgG} 4^{+}$progressively transformed germinal centers showed a markedly elevated IgG4 ${ }^{+} / \mathrm{IgG}^{+}$ plasma cell ratio compared with those with IgG4 ${ }^{-}$ progressively transformed germinal centers (mean $57 \%$ vs $5 \%, P<0.0001)$. Patients with $\mathrm{IgG}^{+}$progressively transformed germinal centers showed an older age distribution and a higher incidence of submandibular lymph node involvement than those with IgG4 ${ }^{-}$progressively transformed germinal centers $(P<0.0001)$.

\section{Pathological Findings in IgG4 ${ }^{+}$Progressively Transformed Germinal Centers}

In patients with IgG4 ${ }^{+}$progressively transformed germinal centers, the lymph nodes demonstrated numerous lymphoid follicles with hyperplastic germinal centers and a distinct mantle zone, but no expansion of the interfollicular zone. Progressively transformed germinal centers were observed in all cases, appearing as round to oval structures 2-3 times the diameter of the other reactive follicles and composed predominantly of small lymphocytes, centrocytes, centroblasts, and numerous mature plasma cells and plasmacytoid cells. The interfollicular zone showed infiltration of a significant number of eosinophils, and T-zones were indistinct (Figure 2).

Interestingly, a unique feature of $\mathrm{IgG} 4^{+}$progressively transformed germinal centers on immunohistochemistry was the localization of the majority of IgG4 ${ }^{+}$plasma cells in the germinal centers, with only a small number present in the interfollicular zone (Figure 2), except in case no. 3, where they were detected in both the germinal centers and interfollicular zone. The IgG4 ${ }^{+}$plasma cells were $>100 / \mathrm{HPF}$ and $\mathrm{IgG}^{+} / \mathrm{IgG}^{+}$plasma cell ratio was $>40 \%$ in all cases (Table 3 and Supplementary Figure 1B). Immunoglobulin light-chain restriction was not detected in any case. These histological and immunohistochemical findings were all compatible with progressively transformed germinal centerstype IgG4-related lymphadenopathy. ${ }^{8,14}$

In contrast, the lymph nodes of patients with IgG4 ${ }^{-}$progressively transformed germinal centers showed heterogenous histological findings, demonstrating a small number or numerous lymphoid follicles with or without hyperplastic germinal centers, and expansion or no expansion of the interfollicular zone. The interfollicular zone did not show a significant number of eosinophils, and T-zones were distinct. IgG4 ${ }^{+}$plasma cells were absent or few, and the $\operatorname{IgG} 4^{+} / \mathrm{IgG}^{+}$plasma cell ratio was $<40 \%$ in all cases.

\section{Disease Progression and Extranodal Lesions in IgG4 Progressively Transformed Germinal Centers}

Thirty-four patients were followed by regular imaging, laboratory findings and clinical evaluation over 3 to 144 months (median, 26 months). During the follow-up period, $23(68 \%)$ patients showed persistence or relapse (or both) of these residual lymph nodes. In all, 18 patients progressed to the development of extranodal lesions, of whom 16 (89\%) interestingly showed the involvement of lacrimal and/or submandibular glands. Moreover, 5 of these 18 patients showed progression to systemic disease (Table 2 and Figure 3; patient nos. 11, 13, 20, 21 and 22). Histologically examined extranodal lesions were consistent with IgG4-related disease.

\section{Clinical Management of IgG4 ${ }^{+}$Progressively Transformed Germinal Centers}

In all, 18 of the 34 patients showed stable disease, despite the presence of persistent residual lymph node lesions in almost all. Ten patients showed the localized or systemic relapse of lymphadenopathy, and were re-biopsied. Nine patients were administered steroid therapy when the lesions progressed, to which all responded well (Table 1). 

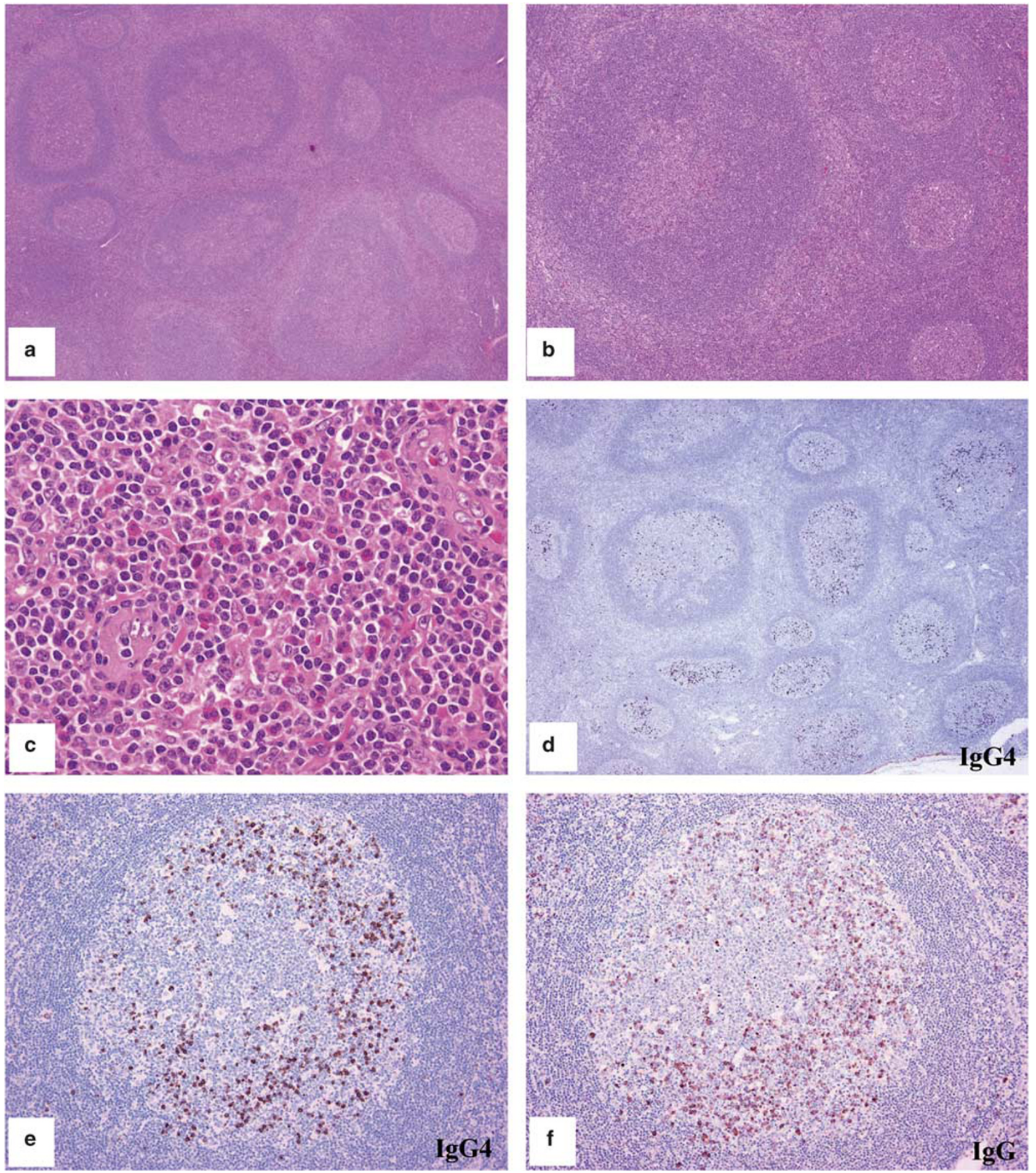

Figure 2 Histological and immunohistochemical features of IgG4 ${ }^{+}$progressively transformed germinal centers. (a, b) Lymph nodes from patient no. 26 showed numerous lymphoid follicles with hyperplasia and progressively transformed germinal centers (hematoxylin and eosin, a: $\times 20$ ). (b) The progressively transformed germinal centers were appearing as round to oval structures 2-3 times the diameter of the other reactive follicles (hematoxylin and eosin, $\times 40$ ). (c) Abundant eosinophil infiltration in the interfollicular zone (hematoxylin and eosin, $\times 200)$. (d) Localization of the majority of IgG4 ${ }^{+}$plasma cells in the germinal centers (IgG4-immunostaining, $\left.\times 20\right) .(\mathbf{e}, \mathbf{f})$ The $\mathrm{IgG}^{+} / \mathrm{IgG}^{+}$plasma cell ratio was $>60 \%$ (IgG4 and IgG-immunostaining, $\times 100$ ). 
a
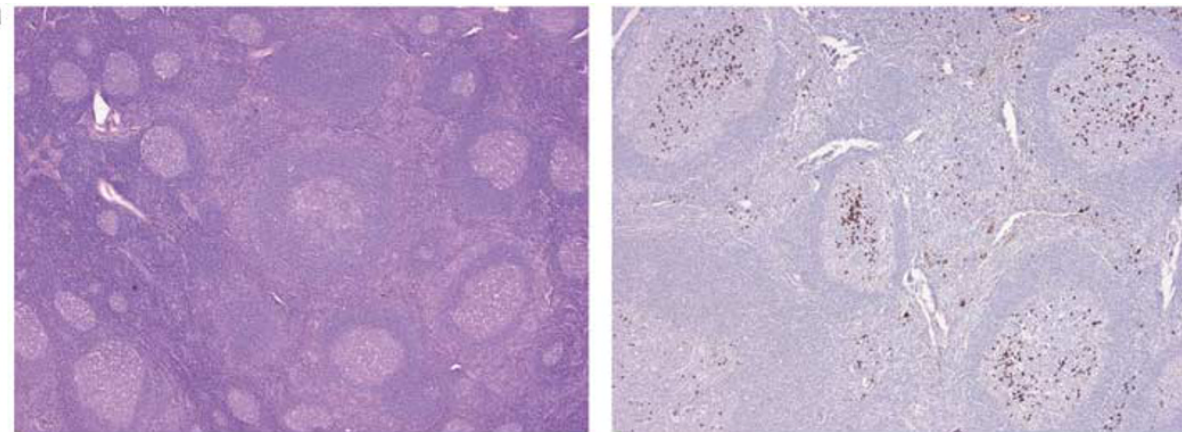

b
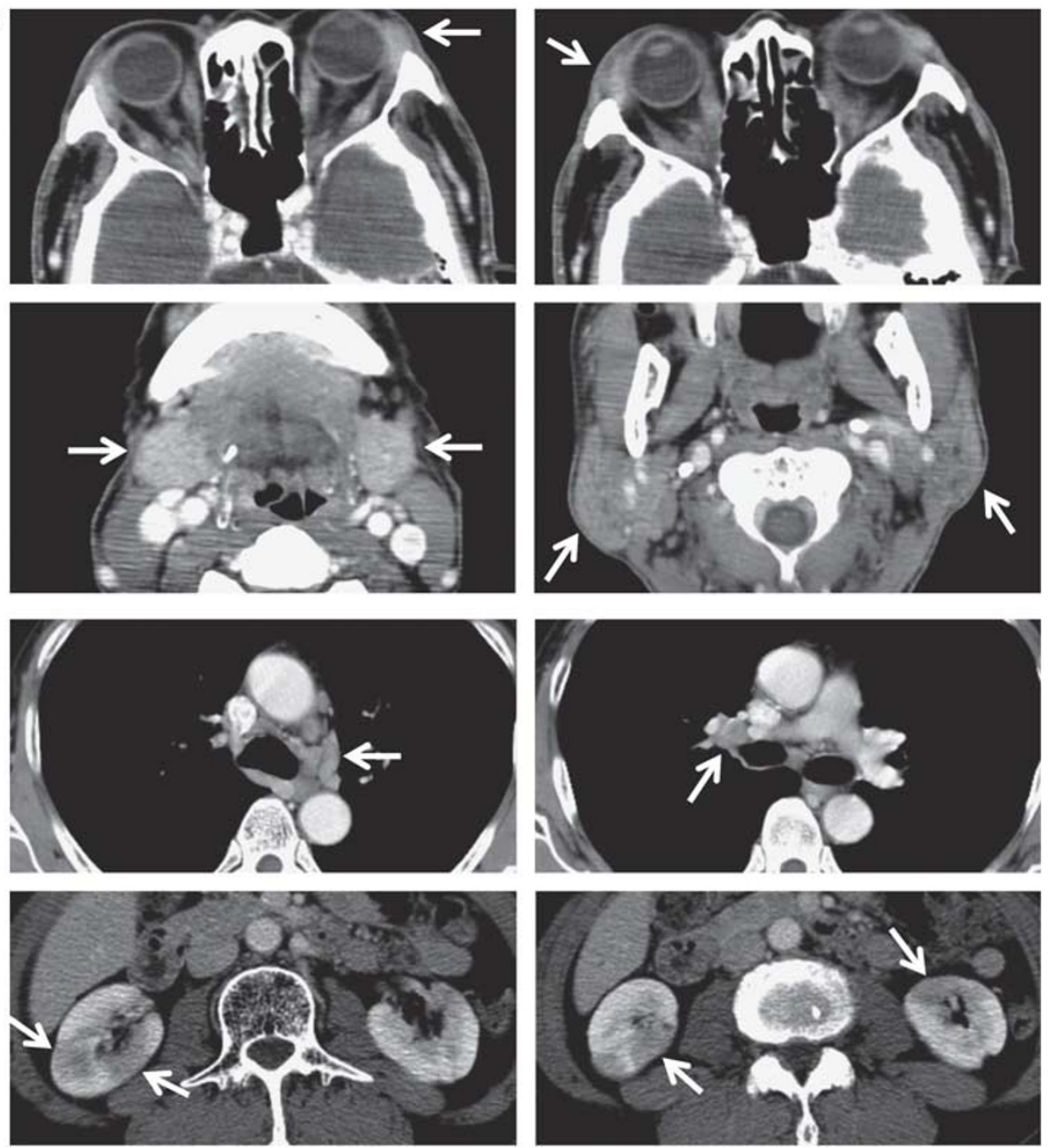

Figure 3 Histological features and radiological images of a patient with IgG4 ${ }^{+}$progressively transformed germinal centers with progression to systemic disease. (a) The affected lymph node from patient no. 11 (initial lymphadenopathy of the submandibular node had been detected 10 years before) showed marked follicular hyperplasia with progressively transformed germinal centers (hematoxylin and eosin, $\times 20$ ). IgG4 ${ }^{+}$ plasma cells were detected in the germinal centers by immunohistochemistry, and the $\operatorname{IgG} 4^{+} / \operatorname{IgG}^{+}$plasma cell ratio was $>40 \%$ (IgG4 immunostaining, $\times 40$ ). (b) The patient showed progression to systemic disease at 10 years after the initial diagnosis. Computed tomography revealed lesions of the lacrimal glands, submandibular glands, parotid glands and kidney, all of which were bilateral, as well as the mediastinum and paraaortic lymph nodes. The lacrimal gland and kidney lesions were histologically consistent with IgG4-related disease, and serum IgG4 levels and IgG4/IgG ratio were highly elevated (serum IgG4, $2260 \mathrm{mg} / \mathrm{dl}$; serum IgG4/IgG ratio, $46 \%$ ). 
Immunoglobulin Heavy-Chain Gene Rearrangement in IgG4 $^{+}$Progressively Transformed Germinal Centers

No immunoglobulin heavy-chain gene rearrangement was observed in any of the cases examined.

\section{Discussion}

In this study, we describe a unique series of 40 patients with progressively transformed germinal centers of the lymph nodes who fulfilled the histological diagnostic criteria of IgG4-related disease. ${ }^{8,10,11-14}$ The disease presented with uniform clinicopathology, namely asymptomatic localized submandibular lymphadenopathy and progression to extranodal lesions, particularly the lacrimal and submandibular glands. Patients were predominantly middle-aged to older males, and about half of those examined had concomitant allergic disease. Microscopic observation of the affected lymph nodes revealed marked follicular hyperplasia with progressively transformed germinal centers, eosinophil infiltration in the interfollicular zone and $\operatorname{IgG} 4^{+}$plasmacytosis in the germinal centers. Eighteen patients developed extranodal lesions, of which those which were histologically examined were consistent with IgG4-related disease. Moreover, all of the examined patients had elevated serum IgG4 and IgE levels, with the exception of three serum IgG4- and one serum IgE-negative patients. These clinicopathological findings of $\operatorname{IgG} 4^{+}$progressively transformed germinal centers are compatible with IgG4-related disease. .10,11-14 $^{8,14}$

IgG4-related disease frequently involves the lacrimal glands, submandibular glands, pancreas, hepatobiliary tract and lymph nodes. ${ }^{7-14}$ Nevertheless, virtually any organ can be affected, including the lungs, mediastinum, skin, retroperitoneum, aorta, kidneys and prostate. ${ }^{-14}$ The general condition of patients at presentation is usually good, with no fever or constitutional symptoms. Common laboratory findings include increased serum IgG4 and IgE levels, whereas lactate dehydrogenase level remains unchanged. Patients often show an excellent response to steroid therapy. ${ }^{7-14}$

Progressively transformed germinal centers is a benign condition of unknown pathogenesis, which presents either as a solitary asymptomatic enlarged lymph node, most commonly in the neck, or in multiple anatomical sites, usually in the form of mass lesions. ${ }^{1-6}$

Progressively transformed germinal centers carries with it an increased long-term risk for the development of nodular lymphocyte predominant Hodgkin lymphoma. ${ }^{3-5}$ However, no case of progression to nodular lymphocyte predominant Hodgkin lymphoma were seen in our available cases.

In the United States and Germany, progressively transformed germinal centers occurs more commonly in young males. ${ }^{1-6}$ Our patients were similar to these previously reported patients in that they were predominantly male and largely presented with solitary asymptomatic lymphadenopathy in the neck, but differed in that they were middle-aged to older. Kojima et $a l^{6}$ also reported that their Japanese progressively transformed germinal centers cases were more frequently middle-aged to older patients, and interestingly that about $30 \%$ had chronic sialadenitis or allergic disease. ${ }^{6}$ These cases are similar to our series of $\mathrm{IgG}^{+}{ }^{+}$progressively transformed germinal centers, suggesting that this clinical picture might be suitably categorized as progressively transformed germinal centers-type IgG4-related lymphadenopathy. In fact, many cases of IgG4-related disease have been reported in Asia, particularly in Japan. ${ }^{8-11}$

Although the mechanism underlying IgG4-related disease remains unclear, ${ }^{8-11}$ a recent study suggested the possible involvement of $\mathrm{T}$ helper 2 cells and regulatory immune reactions, indicating a possible allergic mechanism. ${ }^{16,17}$ In fact, we found elevated serum IgE levels in almost all patients examined. Furthermore, about half of our patients showed eosinophilia, with marked eosinophil infiltration in the affected tissue, in addition to concomitant allergic disease.

Interestingly, our series of IgG4 ${ }^{+}$progressively transformed germinal centers of the lymph nodes appeared to specifically involve the submandibular lymph nodes, but the reason for this is unclear. These nodes receive lymph from a wide area, including the ocular region, nose and adjacent cheek, paranasal sinus, oral cavity, and salivary glands. ${ }^{18}$ This area, particularly the ocular adnexa and salivary glands, is very frequently affected in IgG4-related disease. Indeed, the extranodal lesions detected in our patients frequently involved the area covered by the submandibular lymph nodes. The mechanism might therefore be related to anatomical lymphatic flow.

Three of our patients had normal serum IgG4 levels. This might have been because the measurement of serum IgG4 in these three patients occurred after biopsy, at which time there were no residual main lesions. In this regard, about $20 \%$ of patients with IgG4-related pancreatitis are negative for serum IgG4..$^{8,19,20}$

Residual lymph node lesions in our series of IgG4 ${ }^{+}$ progressively transformed germinal centers patients showed frequent persistence or relapse (or both), and the disease progressed to either or both extranodal lesions or systemic disease. This explains why, although progressively transformed germinal centers was eventually diagnosed based on histological findings, this pattern of disease progression suggested malignant lymphoma.

In conclusion, we describe here a unique case series characterized by progressively transformed germinal centers with intra-germinal center IgG4 ${ }^{+}$ plasmacytosis involving the submandibular lymph node in middle-aged to older patients who clinically 
presented with asymptomatic localized lymphadenopathy. About half of these patients progressed during the follow-up period to extranodal lesions, systemic disease or both. We suggest that the patients with $\mathrm{IgG}^{+}$progressively transformed germinal centers of the lymph nodes may phenotypically present with incipient lesions associated with IgG4related disease. Moreover, almost all cases described here were suspected to be malignant lymphomas at initial diagnosis or when the disease progressed. Prevention of potentially harmful misdiagnosis requires the recognition of this lesion as a distinct clinicopathological entity, based on careful analysis of clinical and pathological findings through the close collaboration of pathologist and clinician.

\section{Acknowledgement}

This work was supported in part by grants from the Okayama Medical Foundation.

\section{Disclosure/conflict of interest}

The authors declare no conflict of interest.

\section{References}

1 Lennert K, Müller-Hermelink HK. Lymphocyten and ihre funktionsformen morphologie, organization und immunologische bedeutung. Verh Anat Ges 1975;69: 19-62.

2 Osborne BM, Butler JJ. Clinical implications of progressive transformation of germinal centers. Am J Surg Pathol 1984;8:725-733.

3 Hansmann ML, Fellbaum C, Hui PK, et al. Progressive transformation of germinal centers with and without association to Hodgkin's disease. Am J Clin Pathol 1990;93:219-226.

4 Osborne BM, Butler JJ, Gresik MV. Progressive transformation of germinal centers: comparison of 23 pediatric patients to the adult population. Mod Pathol 1992;5:135-140.

5 Jones D. Dismantling the germinal center: comparing the processes of transformation, regression, and fragmentation of the lymphoid follicle. Adv Anat Pathol 2002;9:129-138.
6 Kojima M, Nakamura S, Motoori T, et al. Progressive transformation of germinal centers: a clinicopathological study of 42 Japanese patients. Int J Surg Pathol 2003;11:101-107.

7 Sato Y, Ohshima K, Ichimura K, et al. Ocular adnexal IgG4-related disease has uniform clinicopathology. Pathol Int 2008;58:465-470.

8 Sato Y, Notohara K, Kojima M, et al. IgG4-related diseases: historical overview and pathology of hematological disorders. Pathol Int 2010;60:247-258.

9 Masaki Y, Dong L, Kurose N, et al. Proposal for a new clinical entity, IgG4-positive multiorgan lymphoproliferative syndrome: Analysis of 64 cases of IgG4-related disorders. Ann Rheum Dis 2009;68:1310-1315.

10 Masaki Y, Kurose N, Umehara H. IgG4-related disease: a novel lymphoproliferative disorder discovered and established in Japan in the 21st century. J Clini Exp Hematopathol 2011;51:13-20.

11 Cheuk W, Chan JKC. IgG4-related sclerosing disease, a critical appraisal of an evolving clinicopathologic entity. Adv Anat Pathol 2010;17:303-332.

12 Stone JH, Khosroshahi A, Hilgenberg A, et al. IgG4related systemic disease and lymphoplasmacytic aortitis. Arthritis Rheumatism 2009;60:3139-3145.

13 Cheuk W, Yuen HKL, Chu SYY, et al. Lymphadenopathy of IgG4-related sclerosing disease. Am J Surg Pathol 2008;32:671-681.

14 Sato Y, Kojima M, Takata K, et al. Systemic IgG4related lymphadenopathy: a clinical and pathologic comparison to multicentric Castleman's disease. Mod Pathol 2009;22:589-599.

15 Grimm KE, Barry TS, Chizhevsky V, et al. Histopathological findings in 29 lymph node biopsies with increased IgG4 plasma cells. Mod Pathol 2012;25: 480-491.

16 Zen Y, Fujii T, Harada K, et al. Th2 and regulatory immune reactions are increased in immunoglobulin G4-related sclerosing pancreatitis and cholangitis. Hepatology 2007;45:1538-1546.

17 Nakashima H, Miyake K, Moriyama $M$, et al. An amplification of IL-10 and TGF- $\beta$ in patients with IgG4-related tubulointerstitial nephritis. Clin Nephrol 2010;73:385-391.

18 Snell RS. Clinical Anatomy for Medical Students. 6th edn. Lippincott Williams \& Wilkins: Philadelphia, 2000 , pp 659.

19 Hamano H, Kawa S, Horiuchi A, et al. High serum IgG4 concentrations in patients with sclerosing pancreatitis. N Engl J Med 2001;344:732-738.

20 Matsubayashi H, Sawai H, Kimura H, et al. Characteristics of autoimmune pancreatitis based on serum IgG4 level. Dig Liver Dis 2011;43:731-735.

Supplementary Information accompanies the paper on Modern Pathology website (http://www.nature.com/ modpathol) 